\title{
Consensus-based perioperative protocols during the COVID-19 pandemic
}

\author{
Praveen V. Mummaneni, MD, MBA, ${ }^{1}$ John F. Burke, MD, PhD, ${ }^{1}$ Andrew K. Chan, MD, ${ }^{1}$ \\ Julie Ann Sosa, MD, MA, ${ }^{2}$ Errol P. Lobo, MD, PhD, ${ }^{3}$ Valli P. Mummaneni, MD, ${ }^{3}$ \\ Sheila Antrum, RN, ${ }^{4}$ Sigurd H. Berven, MD, ${ }^{5}$ Michael S. Conte, MD, ${ }^{2,6}$ \\ Sarah B. Doernberg, MD, MAS, ${ }^{7,14}$ Andrew N. Goldberg, MD, MSCE, ${ }^{8}$ \\ Christopher P. Hess, MD, PhD, ${ }^{9}$ Steven W. Hetts, MD, ${ }^{9,10}$ S. Andrew Josephson, MD, ${ }^{11}$ \\ Maureen P. Kohi, MD, ${ }^{9,12}$ C. Benjamin Ma, MD, ${ }^{5}$ Vaikom S. Mahadevan, MD, ${ }^{13,14}$ \\ Annette M. Molinaro, PhD, ${ }^{1}$ Andrew H. Murr, MD, ${ }^{8}$ Sirisha Narayana, MD, ${ }^{14}$ John P. Roberts, MD, ${ }^{2}$ \\ Marshall L. Stoller, MD, ${ }^{15}$ Philip V. Theodosopoulos, MD, ${ }^{1}$ Thomas P. Vail, MD, ${ }^{5}$ \\ Sandra Wienholz, RN, ${ }^{16}$ Michael A. Gropper, MD, PhD, ${ }^{3}$ Adrienne Green, MD, ${ }^{14}$ \\ and Mitchel S. Berger, MD'
}

\begin{abstract}
Departments of ${ }^{1}$ Neurological Surgery, ${ }^{2}$ Surgery, ${ }^{3}$ Anesthesia and Perioperative Medicine, ${ }^{5}$ Orthopedic Surgery, ${ }^{8}$ Otolaryngology Head and Neck Surgery, ${ }^{9}$ Radiology and Biomedical Imaging, ${ }^{11}$ Neurology, ${ }^{14}$ Medicine, and ${ }^{15}$ Urology; Divisions of ${ }^{6}$ Vascular and Endovascular Surgery, ${ }^{7}$ Infectious Diseases, ${ }^{10}$ Interventional Neuroradiology, ${ }^{12}$ Vascular and Interventional Radiology, and ${ }^{13}$ Cardiology; ${ }^{4}$ Chancellor's Cabinet; and ${ }^{16}$ Perioperative Care, University of California, San Francisco, California
\end{abstract}

OBJECTIVE During the COVID-19 pandemic, quaternary-care facilities continue to provide care for patients in need of urgent and emergent invasive procedures. Perioperative protocols are needed to streamline care for these patients notwithstanding capacity and resource constraints.

METHODS A multidisciplinary panel was assembled at the University of California, San Francisco, with 26 leaders across 10 academic departments, including 7 department chairpersons, the chief medical officer, the chief operating officer, infection control officers, nursing leaders, and resident house staff champions. An epidemiologist, an ethicist, and a statistician were also consulted. A modified two-round, blinded Delphi method based on 18 agree/disagree statements was used to build consensus. Significant disagreement for each statement was tested using a one-sided exact binomial test against an expected outcome of $95 \%$ consensus using a significance threshold of $p<0.05$. Final triage protocols were developed with unblinded group-level discussion.

RESULTS Overall, 15 of 18 statements achieved consensus in the first round of the Delphi method; the 3 statements with significant disagreement $(p<0.01)$ were modified and iteratively resubmitted to the expert panel to achieve consensus. Consensus-based protocols were developed using unblinded multidisciplinary panel discussions. The final algorithms 1) quantified outbreak level, 2) triaged patients based on acuity, 3) provided a checklist for urgent/emergent invasive procedures, and 4) created a novel scoring system for the allocation of personal protective equipment. In particular, the authors modified the American College of Surgeons three-tiered triage system to incorporate more urgent cases, as are often encountered in neurosurgery and spine surgery.

CONCLUSIONS Urgent and emergent invasive procedures need to be performed during the COVID-19 pandemic. The consensus-based protocols in this study may assist healthcare providers to optimize perioperative care during the pandemic.

https://thejns.org/doi/abs/10.3171/2020.6.SPINE20777

KEYWORDS COVID-19; coronavirus disease 19; surgical triage; perioperative care; infection

ABBREVIATIONS ACS = American College of Surgeons; $I C U=$ intensive care unit; MDPC = multidisciplinary perioperative committee; $P A P R=$ powered air-purifying respirator; PPE = personal protective equipment; PUI = inpatients under investigation; SARS-CoV-2 = severe acute respiratory syndrome coronavirus 2 ; UCPS = UCSF COVID19 PPE Score; UCSF = University of California, San Francisco.

SUBMITTED May 2, 2020. ACCEPTED June 1, 2020.

INCLUDE WHEN CITING Published online October 2, 2020; DOI: 10.3171/2020.6.SPINE20777. 
$\mathrm{H}$ EALTHCARE providers and hospitals face unprecedented challenges during the COVID-19 pandemic. ${ }^{1,2}$ Providers at tertiary-care hospitals in severely affected areas around the world (including those from China, ${ }^{2}$ Taiwan, ${ }^{3}$ Singapore, ${ }^{4}$ and Italy ${ }^{5,6}$ ) have published institutional protocols to help aid healthcare workers in this crisis. ${ }^{7-9}$ As the pandemic has now spread to the US, ${ }^{10,11}$ it is imperative to adapt these algorithms to the US healthcare setting. ${ }^{12}$ The University of California, San Francisco (UCSF) is a quaternary-care academic hospital that treats patients primarily from California and the Western US. During the California shelter-in-place order, patients who need urgent and emergent invasive procedures continue to seek medical attention through telehealth clinics, emergency department visits, or direct inpatient transfers to our institution. Standardized protocols are necessary to triage patients for urgent/emergent procedures and surgeries in the setting of decreased capacity due to a shortage of staffed beds as well as limited personal protective equipment (PPE), blood bank supplies, and ventilators. Furthermore, guidance is needed to minimize nosocomial transmission of COVID-19 to patients and staff during invasive procedures. ${ }^{13}$

There is insufficient data and time in the midst of a pandemic to create evidence-based guidelines for triaging invasive procedures. ${ }^{14}$ Thus, we present our institutional experience using a modified Delphi method. In brief, the Delphi method consists of first forming an expert panel, creating a series of statements that are submitted to the panel, obtaining anonymous feedback from the panel, amending the statements, and iterating this process until consensus is achieved. Here, we use this method to create consensus-based procedural triage protocols and checklists during the COVID-19 pandemic.

\section{Methods}

A multidisciplinary perioperative committee (MDPC) was tasked with the creation of a triage system and management protocols for patients requiring urgent and emergent procedures and surgeries during the COVID-19 pandemic. The MDPC is composed of 26 leaders from 10 academic departments, including 7 department chairpersons, the chief medical officer, the chief operating officer, infection control officers, nursing leaders, and resident house staff champions. The departments involved included anesthesiology, cardiology (interventional team), internal medicine (hospitalist and infectious disease teams), neurology (stroke and neurocritical care team), neurosurgery, orthopedics, otolaryngology-head and neck surgery, radiology (interventional and neurointerventional teams), surgery (including cardiothoracic, general, pediatric, pediatric cardiac, plastic, transplant, and vascular surgery), and urology. The MDPC identified barriers to the care of patients needing invasive procedures, including the availability of PPE, ventilators, intensive care unit (ICU) and floor beds, blood bank resources, and nursing and physician staffing shortages.

We used a modified Delphi method to build consensus within the MDPC expert panel to expedite the creation of urgently needed perioperative protocols and checklists. ${ }^{15,16}$ A series of 18 statements were created that synthesized recommendations from the WHO, Centers for Disease Control and Prevention, ${ }^{17}$ American College of Surgeons (ACS), ${ }^{18}$ UCSF institutional policies, and San Francisco Department of Public Health order no. C19-07 ("shelter in place"). The 18-item questionnaire was sent to the expert panel in the form of agree/disagree statements. ${ }^{16}$ Experts were blinded to each other's responses.

\section{Statistical Analysis}

Statistically, the statements were created with an expectation of consensus (i.e., agreement of $\geq 95 \%$ ); we tested this expected outcome using a one-sided exact binomial test. Statistically significant disagreement was defined using a $\mathrm{p}$ value threshold of 0.05 . Items with statistically significant disagreement were further investigated through direct communication with individual members of the expert panel. In the second round of the Delphi method, we modified statements with significant disagreement and resubmitted the amended statements in a blinded fashion to the expert panel. This iterative process was continued until there was no statistically significant disagreement for any statement.

After the iterative, blinded questionnaires were completed, we unblinded the expert panel and sought feedback using both direct (in-person videoconferencing) and indirect (group e-mail) communication methods. At this point, we included input from a statistician, an epidemiologist, and an ethicist, who provided consultation on the final protocol but did not participate in the Delphi questionnaire. The total time to complete the Delphi approach was 14 days, including 10 days to obtain blinded feedback on statements and 4 days to obtain universal agreement on the finalized protocols and checklists.

\section{Results}

From the MDPC, we received responses from 26 members (100\% response rate) to the blinded questionnaire. In Fig. 1, we plot the percentage agreement for each item of the questionnaire, grouped by category. Three statements regarding case acuity, case triaging, and use of PPE showed statistically significant disagreement $(\mathrm{p}=$ $0.009, p<0.001$, and $p=0.009$, respectively). The second round of the blinded Delphi questionnaire included three modified agree/disagree statements on these controversial topics, which subsequently met a priori criteria for consensus (no statistically significant disagreement; $p$ $>0.05$ for all statements). After two rounds of blinded questionnaires were completed by panel members, a videoconference meeting was held for direct, group-level feedback (our institution has discouraged large, in-person physical gatherings during the pandemic). Furthermore, e-mail was used to disseminate and receive feedback on the finalized protocols and checklists (Figs. 2-4). The final protocols 1) quantified outbreak level, 2) categorized patient acuity, 3) provided a checklist for urgent/emergent procedures, and 4) created a novel scoring system for the allocation of limited PPE. 


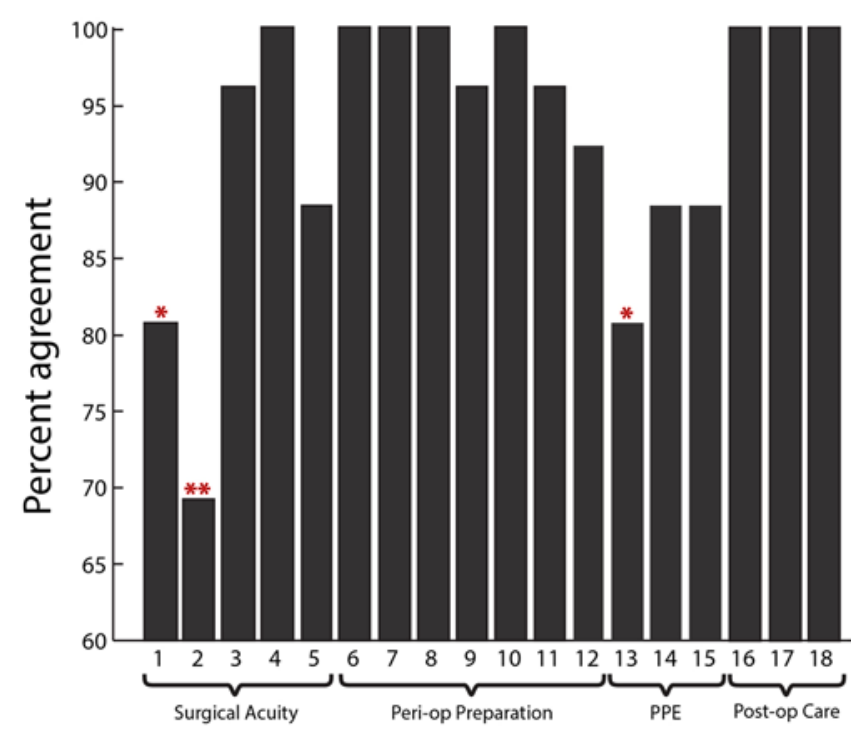

Delphi statement number

FIG. 1. Delphi method results. The statements are listed on the x-axis, and the percentage agreement of each statement after the first round of the Delphi questionnaire is shown on the y-axis. Significant disagreement is shown with an asterisk $\left({ }^{*} p<0.01,{ }^{* *} p<0.001\right)$. Statements are grouped by categories, including case acuity, perioperative preparations, PPE, and postoperative care. Figure is available in color online only.

\section{Triaging Invasive Procedures}

In Fig. 2, we present our hospital's COVID-19 invasive procedure triage algorithm. The algorithm is based on quantifying both the hospital's COVID-19 patient surge status and the acuity of patients needing an invasive procedure. The COVID-19 patient surge status is represented by a four-tiered, color-coded scale characterized as follows: 1) green level, $\leq 1 \%$ total inpatients under investigation (PUI) for COVID-19 infection or confirmed COVID-19 positive (COVID+); 2) yellow level, 1\%-5\% total PUI/COVID+ inpatients or up to $20 \%$ staffing shortages related to the pandemic; 3 ) red level, $>5 \%$ of total PUI/COVID+ inpatients or $>20 \%$ staffing shortages; and 4) black level, overwhelming number of COVID+ patients inundating the capacity of the medical center (e.g., no available ICU ventilators), thereby creating the need for nearby field hospitals to take patient overflow. Of note, the percentage of PUI/COVID+ inpatients is expressed as the number of COVID-19 patients in the hospital divided by the total number of beds multiplied by $100 \%$. Defining surge levels by both the number of COVID+ patients and the overall hospital capacity allows for more flexibility to manage surgical and invasive procedures.

Our case-acuity algorithm is based on the three-tiered ACS recommendations..$^{18}$ In addition, using our Delphi system, we developed subcategories within this tiered sys-

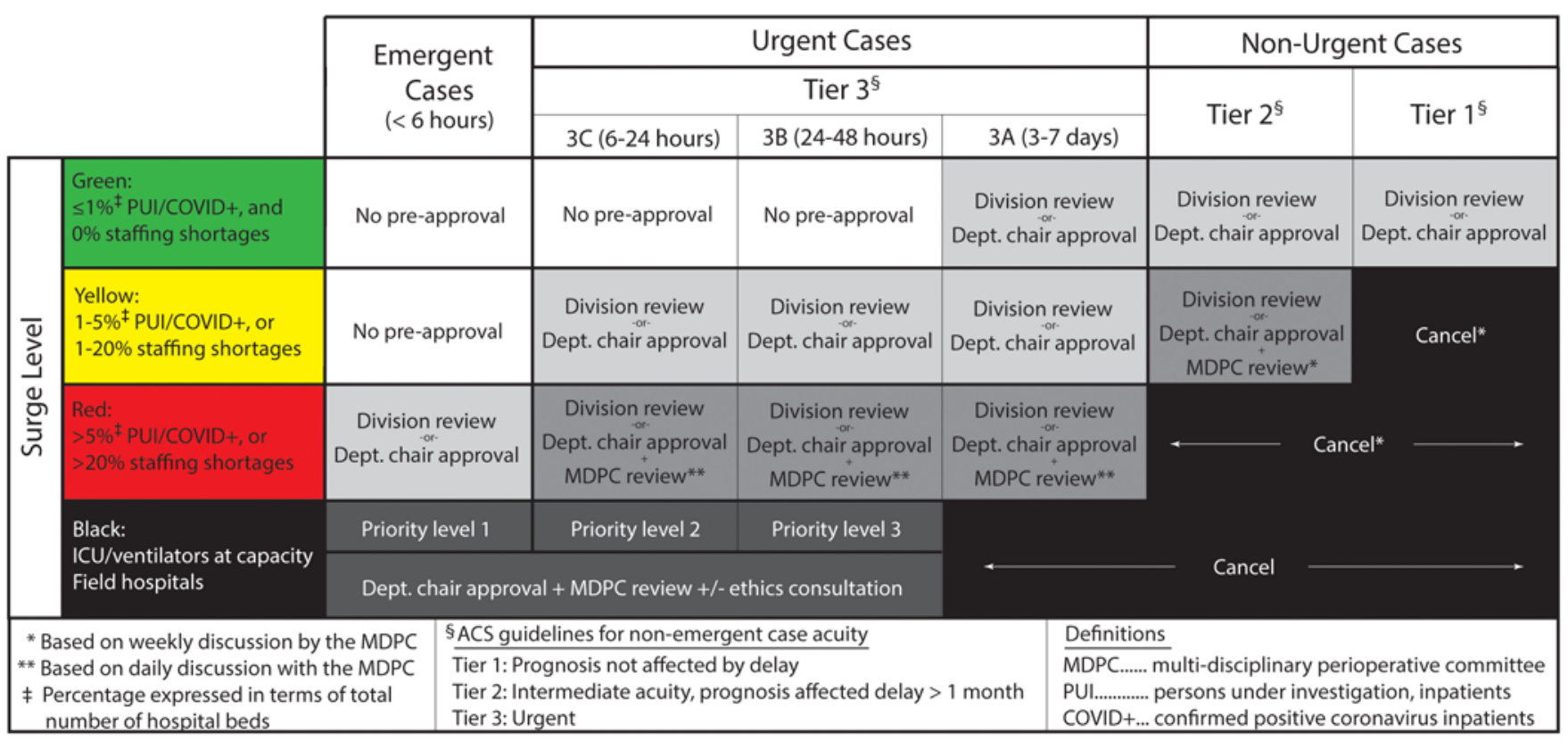

FIG. 2. Surgical and invasive procedural triage algorithm. The hospital capacity criteria are shown in the left column. In the green level, $\leq 1 \%$ of admitted patients are under investigation for COVID-19 infection or are confirmed COVID-19 positive (COVID+). In the yellow level, $1 \%-5 \%$ PUI/COVID+ inpatients or up to $20 \%$ staffing shortages are present. In the red level, $>5 \%$ or more PUI/COVID+ inpatients or $>20 \%$ staffing shortages are present. In the black level, an overwhelming number of COVID+ patients inundate the capacity of the medical center (e.g., no available ICU ventilators). The case acuity is shown on the top row and is based on the three-tiered ACS recommendations. Tier 1 cases represent low-acuity cases that can be postponed (elective); tier 2 cases represent intermediate-acuity cases that are not urgent; and tier 3 cases represent high-acuity urgent cases. Urgent (tier 3) cases were further subcategorized by consensus of the MDPC: $3 A$ cases require intervention within 1 week, 3B cases require intervention within 48 hours, and $3 \mathrm{C}$ cases require intervention within 24 hours. Emergent cases must be undertaken within 6 hours if resources are available to protect a patient's life or to prevent devastating morbidity. In the algorithm, the ability for a case to proceed (and the need for preapproval) is shown for all combinations of hospital capacity and case acuity. 


\begin{tabular}{|c|c|}
\hline Category & Point Value \\
\hline \multicolumn{2}{|l|}{ 1. Surgical Risk } \\
\hline High: $\begin{array}{l}\text { Surgery involving potential } \\
\text { aerosolization of SARS-CoV-2 }\end{array}$ & 2 \\
\hline Intermediate: General anesthesia & 1 \\
\hline Low: Local anesthesia & $\mathbf{0}$ \\
\hline \multicolumn{2}{|l|}{ 2. COVID+ Status } \\
\hline Confirmed COVID-19 infection & 2 \\
\hline Suspected COVID-19 infection & 1 \\
\hline Asymptomatic Patient & 0 \\
\hline \multicolumn{2}{|l|}{ Point Total } \\
\hline $\begin{array}{r}\mathrm{N} 95 / \mathrm{PA} \\
\text { (If not available, case rec }\end{array}$ & $\begin{array}{c}\text { N95/PAPR required } \\
\text { (If not available, case requires consultation with MDPC) }\end{array}$ \\
\hline N95/PAPR & N95/PAPR recommended \\
\hline \multicolumn{2}{|c|}{$\begin{array}{l}\text { Standard surgical PPE } \\
\text { (May use N95/PAPR if available) }\end{array}$} \\
\hline
\end{tabular}

FIG. 3. The UCPS. The scoring system is used to allocate our limited supply of PPE based on procedural and patient-related risks for viral transmission. For a UCPS score of $0-1$, only standard surgical PPE is required. For a UCPS score of 2, an N95 or PAPR mask is recommended, and for a UCPS score of 3 or 4 , an N95 and/or PAPR mask is mandatory.

tem to permit more granular, time-based triaging. Using ACS guidelines, tier 1 cases represent low-acuity cases that can be postponed (elective), tier 2 cases represent intermediate-acuity cases that are not urgent, and tier 3 cases represent high-acuity, urgent cases that should proceed during the COVID-19 pandemic if capacity allows. Urgent (tier 3) cases were further subcategorized by consensus of the MDPC: $3 \mathrm{~A}$ cases require intervention within 1 week, 3B cases require intervention within 48 hours, and $3 \mathrm{C}$ cases require intervention within 24 hours. Finally, there are emergent cases that must be undertaken as soon as possible (within 6 hours) to protect a patient's life or limb or to prevent devastating morbidity. Many neurosurgical and spine procedures fall into the third tier. For example, an acute spinal cord injury with cord compression would be a tier $3 \mathrm{C}$ case $(<24$ hours), a spinal tumor with a stable neurological deficit would be a tier $3 \mathrm{~B}$ case ( $<48$ hours), and a herniated disc with a foot drop would be a tier $3 \mathrm{~A}$ case $(<1$ week).

Combining outbreak burden and case acuity resulted in the triage system in Fig. 2. Specifically, in the green level, cases that are classified as emergencies and tier 3B/C can be booked without preapproval. However, tier 1, 2, and $3 \mathrm{~A}$ cases require review from the division chief/department chair. In the yellow level, emergent cases require no preapproval, but all tier 3 cases require approval from the division chief/department chair. Furthermore, tier 1 and 2 cases require approval from the division chief/department chair with input from members of the MDPC. In the red level, emergent cases require approval from the division chief/department chair, all tier 3 cases require approval from the division chief/department chair and input from members of the MDPC, while tier 1 and 2 cases are cancelled. In the black level, tier 1,2, and $3 \mathrm{~A}$ cases are cancelled, and tier $3 \mathrm{~B}, 3 \mathrm{C}$, and emergent cases must be approved by the department chair and must be allowed by the MDPC. Finally, if the hospital reaches the black level, the MDPC is encouraged to seek the guidance of an ethicist if ICU beds and ventilators need to be rationed between patients. ${ }^{19}$ There may be situations in the black level when medical providers need to decide whether a COVID-19-infected patient is assigned the last ventilator or a patient in need of an emergency lifesaving procedure should be allotted the last ventilator.

\section{Surgical COVID-19 Transmission Risk and Use of PPE}

Once the decision is made to schedule a case, our Delphi system identified two groups needing PPE during invasive procedures: one group is the anesthesia team, who are at risk of coronavirus transmission during airway access, and the other group includes the proceduralists/surgeons, nursing staff, and procedural ancillary technicians.

The MDPC categorized all intubation/extubation events as having the potential to aerosolize severe acute respiratory syndrome coronavirus 2 (SARS-CoV-2) from the airway. Consequently, our protocol requires that only anesthesia staff remain in the room during intubation and extubation. The proceduralists/surgeons and nurses/staff should wait outside the procedure room for 15 minutes after the completion of airway access. The laminar airflow was calculated within the operating rooms to determine that 15 minutes is adequate for safe return of proceduralists and nurses. During airway access and the 15-minute airway pause, the anesthesia team is required to wear N95 and/or powered air-purifying respirator (PAPR) masks, and at least one representative from nursing and the procedural/surgical team are required to remain within visual sight or audible distance of the anesthesia team.

Outside of airway access, PPE requirements for physicians and staff are based on the UCSF COVID-19 PPE score (UCPS; Fig. 3), which we developed to allocate our limited PPE (N95/PAPR masks). This scoring system was discussed with our infection control team and is based on grading procedural and patient risk factors for SARS$\mathrm{CoV}-2$ transmission to healthcare providers. Of note, this score is implemented only if access to PPE (N95/PAPR) is limited. If a hospital has a sufficient supply of N95/PAPR, it is reasonable to make PPE available for every suspected COVID case. However, many hospitals (including our own) did not have access to unlimited N95/PAPR, and this score was developed as a method of allocating scarce PPE under these conditions.

Cases with low transmission risk (level 0) are defined as nonaerosolizing procedures that do not require general anesthesia (such as cardiac catheterization); intermediaterisk cases (level 1) are defined as nonaerosolizing procedures that require general anesthesia (such as lowerextremity vascular bypass surgery); and high-risk cases (level 2) are procedures with the potential to aerosolize the virus during the case, including craniofacial surgery, 


\section{Checklist for Perioperative Care During COVID-19 Pandemic Preoperative checklist}

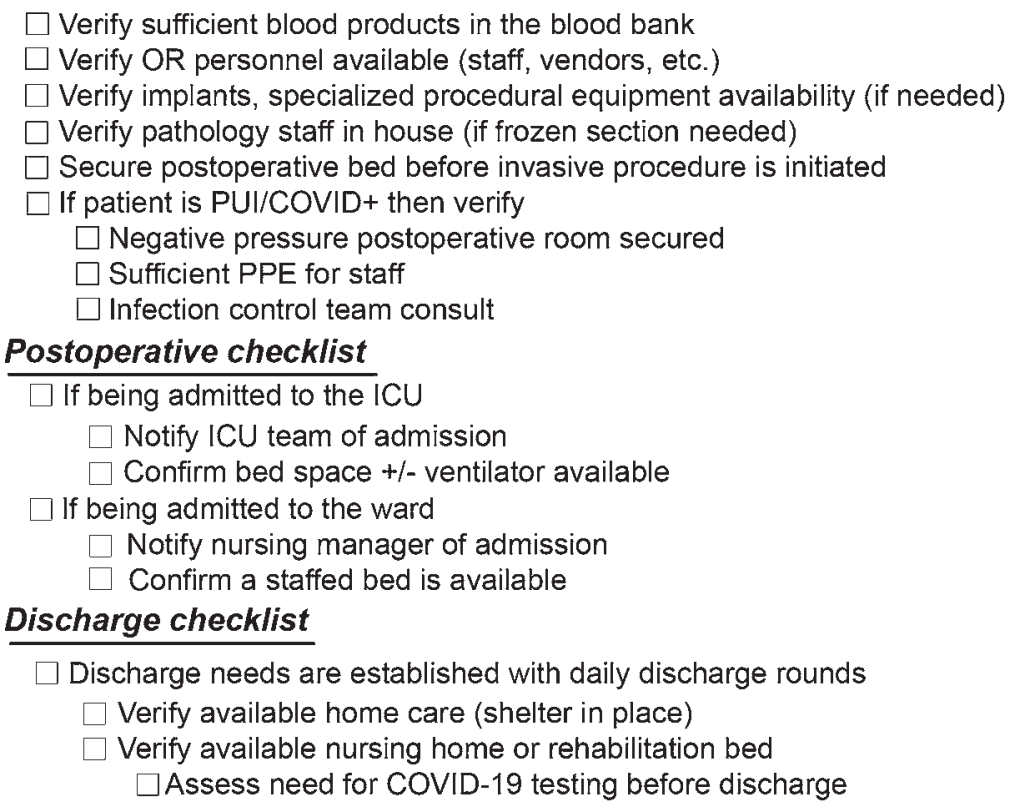

FIG. 4. Checklist for perioperative care during the COVID-19 pandemic. OR = operating room.

sinus surgery, and anterior skull base surgery. For patient transmission risk, low-risk patients (level 0) have no symptoms of COVID-19 infection or have tested negative for COVID-19 infection, intermediate-risk patients (level 1) have suspected (respiratory symptoms) but not confirmed COVID-19 infection, and high-risk patients (level 2) have tested positive for COVID-19 infection. As there is currently a lag with COVID-19 test results at UCSF, it is possible that COVID-19 testing will not be completed in time for a patient who needs an urgent/emergent procedure.

Summing these procedural and patient risk levels yields the UCPS (Fig. 3). For a UCPS of $0-1$, only standard surgical PPE is required. For a UCPS of 2, an N95 or PAPR mask is recommended, and for a UCPS of 3 or 4 , an N95 and/or PAPR mask is mandatory. If N95/PAPR masks are not available for a UCPS 3 or 4 case, then the proceduralist/surgeon should consult with the MDPC and the case may be cancelled to protect the healthcare team.

\section{Preoperative, Perioperative, and Postoperative Care}

We developed a checklist to ensure safe and effective perioperative care. Before any invasive procedure is performed, the checklist verifies that a staffed bed will be available for the patient following the procedure (Fig. 4). This checklist also verifies ahead of time that sufficient blood bank supply, pathology specimen processing, necessary implants, and specialized procedural equipment (such as endoscopes and microscopes) are available. If any of these items are needed but their availability is not verified preoperatively, we recommend delaying or aborting the procedure. The checklist also addresses the need for negative-pressure rooms and verification of PPE on the ward or ICU based on COVID-19 status.
For postoperative care, we verify that both a staffed bed and, if needed, a ventilator are available. In addition, the checklist ensures that the ICU and/or nursing teams taking care of the patient after the procedure receive direct, personal sign-out from the proceduralists/surgeons and anesthesia staff. For discharge planning, the discharge planner must assess the need for COVID-19 testing prior to placement in skilled nursing or rehabilitation facilities as well as bed availability at these institutions. If a patient is to be discharged home, then family or home care should be available to help patients adhere to shelter-in-place instructions from state and local officials. Discharge planning requires close attention as unanticipated increases in hospital length of stay will place excess strain on hospital capacity postoperatively in the event of disease surge.

\section{Case Illustration}

The patient is a 63-year-old man with prostate cancer, a known T11 metastatic tumor, and failed radiotherapy to the lesion, who presented with severe thoracic back pain and inability to ambulate over 1 week. He denied recent fevers, chills, upper respiratory symptoms, sore throat, or known COVID-19+ contacts. On telehealth examination, his motor examination revealed weakness in his right lower extremity. MRI revealed a T11 epidural metastatic lesion resulting in significant cord compression (Fig. 5A and B). CT revealed a lytic lesion associated with T11 and T12 pathologic fractures, segmental kyphosis, 25\%-50\% collapse of the vertebral body, and involvement of bilateral posterior spinal elements (Fig. 5C and D). Laboratory values were notable for a hemoglobin level of $9 \mathrm{~g} / \mathrm{dl}$.

Applying the UCSF surgical protocol (Fig. 3), the hospital was investigating 7-24 PUI/COVID+ patients, yield- 

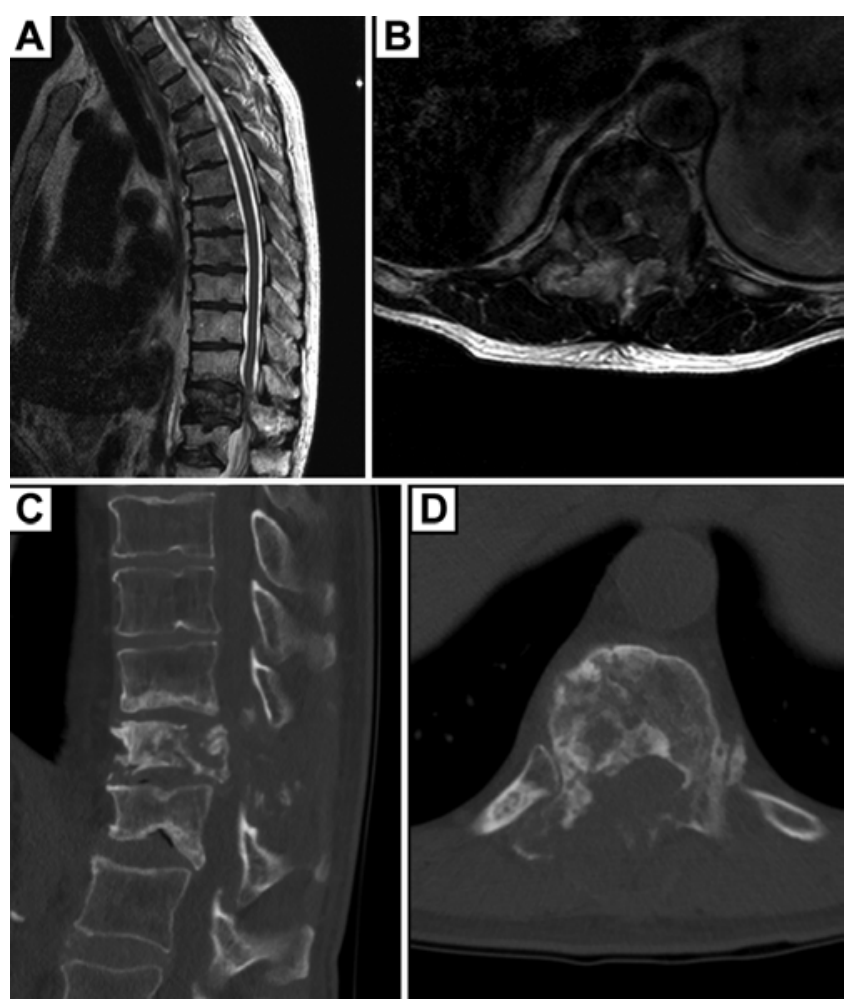

FIG. 5. Preoperative sagittal (A) and axial (B) MR images demonstrating a T11 epidural metastatic lesion and significant cord compression. Preoperative sagittal (C) and axial (D) CT images demonstrating a lytic T11 lesion associated with T11 and T12 pathologic fractures, segmental kyphosis, and 25\%-50\% collapse of the vertebral body. Bilateral involvement of the posterior elements was noted. Given the cord compression, this procedure was graded as a tier $3 \mathrm{~B}$ case (needs to be addressed within 48 hours) and was completed when the hospital was in the yellow level; thus, it was agreed to proceed immediately to surgery (see Fig. 2).

ing a yellow surge level. The case urgency-dictated by the rapidly progressive inability of the patient to ambulate and a Spinal Instability Neoplastic Score ${ }^{20}$ of $15(\geq 13$ unstable)—was deemed such that the case should proceed within 48 hours, categorizing the surgery as tier $3 \mathrm{~B}(<48$ hours; Fig. 3). Therefore, department chair approval in the context of weekly discussion by the MDPC was required and obtained.

The triage score was 1 , as the surgery received 1 point for general anesthesia (intermediate surgical risk) and 0 points for the patient's lack of COVID-19 symptoms (asymptomatic). Therefore, the surgery proceeded using standard surgical PPE.

Operatively, the patient received a minimally invasive T11 laminectomy and transpedicular approach for tumor resection with concomitant stabilization with T9-L1 percutaneous screw placement and instrumented fusion (Fig. 6 ). The minimally invasive approach was chosen 1) to reduce blood loss in this anemic patient because of the limited blood bank product availability during COVID-19, and 2) to decrease potential wound-healing issues, including surgical site infection, given the patient's history of prior radiation and preference to avoid in-person postoperative
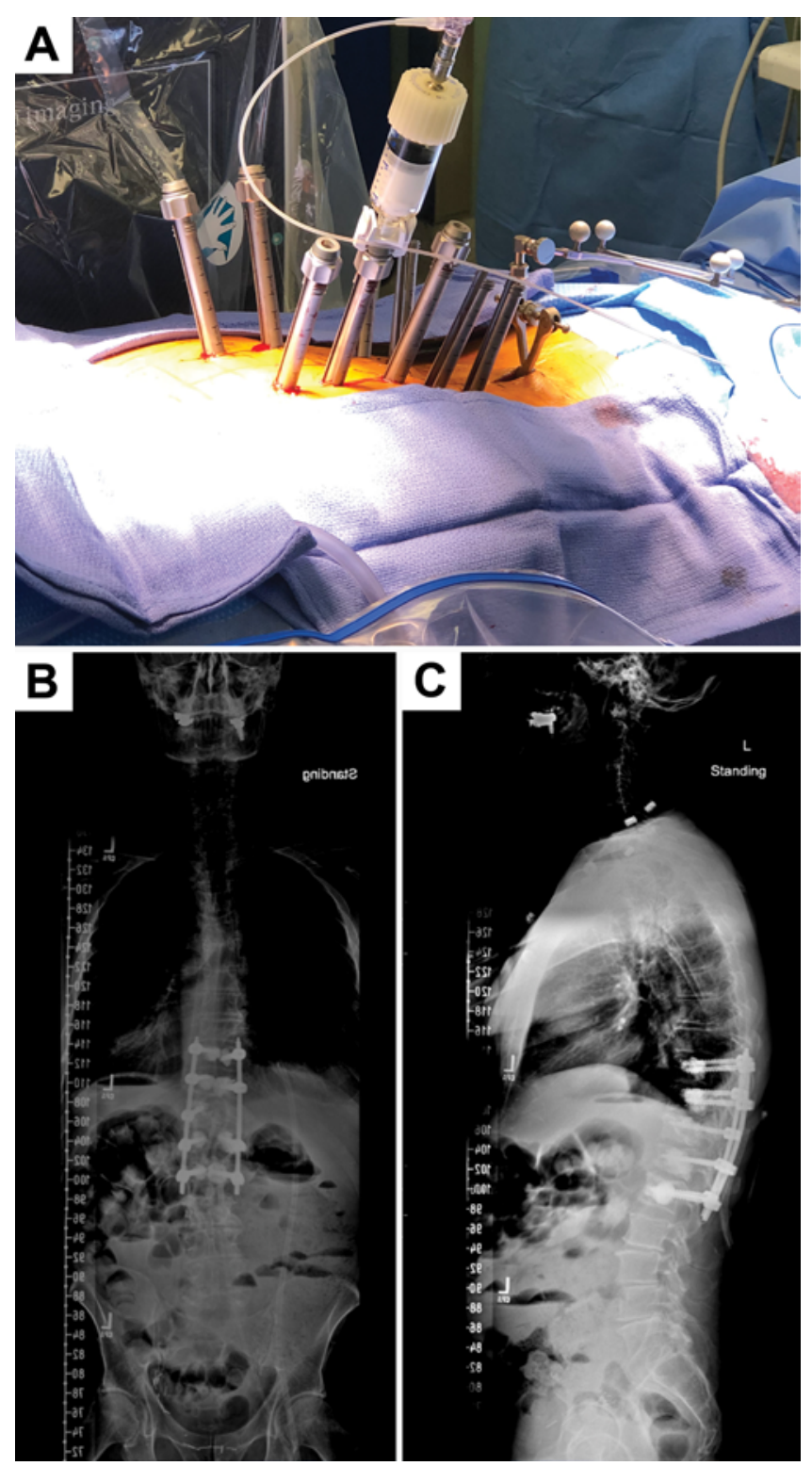

FIG. 6. Intraoperative image (A) showing the small minimally invasive incisions for percutaneous pedicle screw placement and a small midline incision overlying the tumor level. This patient had prior radiotherapy, so small incisions were utilized to avoid wound-healing problems and limit blood loss. Postoperative anteroposterior (B) and lateral (C) standing plain radiographs showing spinal instrumentation in place. Figure is available in color online only.

visits and readmissions that may place the patient at increased risk of nosocomial infection with COVID-19. His motor examination improved to full strength in the bilateral lower extremities when he was discharged home on postoperative day 5 with pain well controlled.

\section{Discussion}

The COVID-19 outbreak, caused by the SARS-CoV-2 virus (Fig. 7), has resulted in rapidly changing and unpredictable strains on the healthcare system. ${ }^{12,21-23}$ Given the 


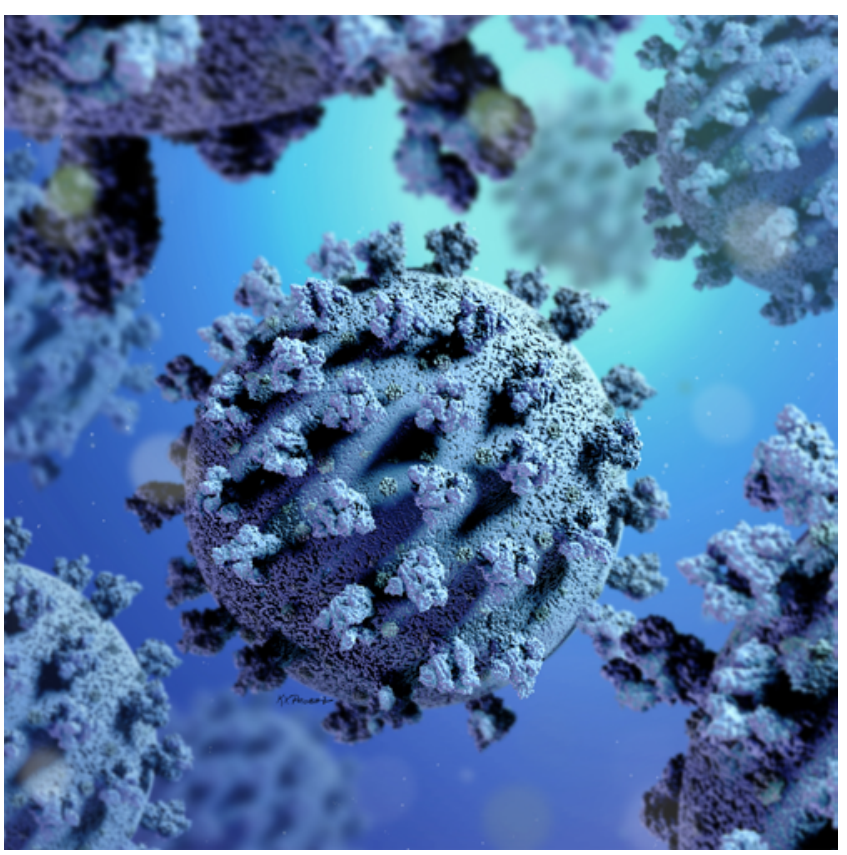

FIG. 7. The SARS-CoV-2 virus responsible for COVID-19. Artist: Kenneth Probst. Copyright UCSF Department of Neurological Surgery. Published with permission.

dynamic nature of the pandemic and the governmental response, ${ }^{17}$ UCSF was in need of urgent protocols and checklists to triage perioperative care for invasive procedures. With insufficient time to create evidence-based guidelines, we turned to a modified Delphi approach to achieve consensus among faculty leaders from 10 departments, infection control experts, hospital administrators, nursing leaders, and resident house staff champions. We also consulted a bioethicist, epidemiologist, and statistician.

We prioritized flexibility in our protocol. Specifically, we do not attempt to define specific case types and situations that mandate urgent versus emergent care; such definitions will vary based on patient-specific factors and the judgment of providers in varied specialties. Instead, Fig. 2 provides a protocol for scheduling cases based on the urgency of the needed procedure as determined by the specialist provider and the department chair, with input from the MDPC. The color-coded outbreak levels help providers triage urgent and emergent cases based on hospital capacity. We avoided triaging cases based on patient comorbidities, ${ }^{24}$ because the COVID-19 pandemic may rapidly alter the health status of patients.

Regarding PPE, there is a limited supply. We created the UCPS scoring system to help guide providers on which form of PPE to use for their cases. If N95/PAPR masks are absent in cases in which the UCPS score is 3 or 4 , we suggest that proceduralists/surgeons consider cancelling the case (independent of acuity) given the risk of SARS-CoV-2 transmission to staff, other patients, and ultimately the public. This issue should be discussed in real time with the MDPC. The UCPS score takes into account the underlying COVID-19 status of the patient. Consistent with this, we have adopted a policy of screening every surgical patient for COVID-19 within 4 days preoperatively. This precaution has minimized the concern of hospital staff about the risk of restarting elective procedures, as well as limited the use of N95/PAPR in cases that are deemed low COVID-19 transmission risk (UCPS of 2 or less). Currently, preoperative COVID-19 testing is readily available for essentially all patients who are candidates for urgent and elective surgery.

Checklists (Fig. 4) help operationalize the preparation and execution of procedures, ${ }^{25,26}$ reduce morbidity and mortality, ${ }^{27}$ and minimize medical error. ${ }^{28}$ During the COVID-19 pandemic, typical care pathways are disrupted, potentially increasing the risk of errors. ${ }^{29}$ Rigorously adhering to checklists helps maintain a culture of safety. ${ }^{30,31}$

Our protocol may be particularly relevant to spinal surgery as the three-tiered $\mathrm{ACS}^{18}$ system for establishing case acuity includes an "urgent" tier 3 category that is ambiguous when applied to spine procedures. Spine surgery involves a wide range of "urgent" cases that may still be further categorized as needing to proceed within 1 week (e.g., herniated disc with foot drop), within 48 hours (such as a spinal tumor with stable neurological deficit), or within 24 hours (e.g., acute spinal cord injury with compression or stenosis with cauda equina syndrome). Our protocol addresses these nuances of spine surgery specifically.

In terms of limitations, largely due to the unprecedented nature of the pandemic, it took 14 days to complete the Delphi process and develop a streamlined perioperative protocol. Our current experience with the Delphi process will likely allow us to create future protocols more expeditiously. One goal of the current protocol, aside from outlining safe practices for conducting invasive procedures during the pandemic, is to provide a blueprint of how surgeons and proceduralists can triage cases in future crises. Having contingency plans in place for such situations can create a culture of perioperative preparedness. Another limitation of the study is that the exact criteria for defining the surge levels (Fig. 2) were estimated by our hospital's epidemiologists. Hopefully, with the benefit of hindsight, future studies will be able to provide more data-driven methods to quantify viral prevalence in the community. Until then, these criteria served to standardize the overall level of strain on our healthcare system, allowing more rapid and effective communication across our institution.

Now that we have weathered the initial outbreak of COVID-19, it is important to document the protocols used by various hospital systems to manage perioperative services through the pandemic. Specifically, COVID-19 is likely to remain in the population moving forward, making it possible for outbreaks to occur in local (or global) populations in the years to come. To prepare for this, it is imperative that hospital systems have a systematic method of triaging urgent and nonurgent invasive procedures, as well as allocating scarce PPE. To that end, the protocols (algorithm, checklist, and UCPS score) presented in Figs. 2-4 provide a principled framework for standardizing the care of patients who need urgent and emergent invasive procedures during the pandemic. In addition, in our own hospital system, we have created several more general interventions to manage potential additional surges once elective cases restart, including creating 1) COVID units (isolated inpatient areas designed to rapidly accommodate 
COVID+ patients), 2) new cleaning protocols, and 3) space restrictions to allow adequate distancing in waiting rooms, among other changes. Finally, when hospital resources are near capacity, we suggest seeking the guidance of a multidisciplinary committee using transparent processes to allocate scarce resources.

\section{Conclusions}

The COVID-19 pandemic poses a significant challenge to the triage and care of patients who need urgent and emergent invasive procedures. There is an immediate need for protocols and checklists in the setting of scarce PPE, limited hospital capacity, and limited staffing. To aid this process, we created consensus-based protocols that we now use to guide perioperative care at UCSF. The protocols assess hospital capacity, triage urgent/emergent cases, implement a perioperative safety checklist, and guide the optimal use of limited PPE through the deployment of a novel scoring system (UCPS). This framework minimizes the strain that urgent/emergent invasive procedures and surgeries place on hospital resources during the pandemic.

\section{References}

1. Wu F, Zhao S, Yu B, et al. A new coronavirus associated with human respiratory disease in China. Nature. 2020;579(7798): 265-269.

2. Zhou F, Yu T, Du R, et al. Clinical course and risk factors for mortality of adult inpatients with COVID-19 in Wuhan, China: a retrospective cohort study. Lancet. 2020;395(10229): 1054-1062.

3. Wang CJ, Ng CY, Brook RH. Response to COVID-19 in Taiwan: big data analytics, new technology, and proactive testing. JAMA. 2020;323(14):1341-1342.

4. Wong JEL, Leo YS, Tan CC. COVID-19 in Singapore-current experience: critical global issues that require attention and action. JAMA. 2020;323(13):1243-1244.

5. Spina S, Marrazzo F, Migliari M, et al. The response of Milan's Emergency Medical System to the COVID-19 outbreak in Italy. Lancet. 2020;395(10227):e49-e50.

6. Grasselli G, Pesenti A, Cecconi M. Critical care utilization for the COVID-19 outbreak in Lombardy, Italy: early experience and forecast during an emergency response. JAMA. 2020;323(16):1545-1546.

7. Guan WJ, Ni ZY, Hu Y, et al. Clinical characteristics of coronavirus disease 2019 in China. N Engl J Med. 2020;382(18): 1708-1720.

8. Hoehl S, Rabenau H, Berger A, et al. Evidence of SARSCoV-2 infection in returning travelers from Wuhan, China. $N$ Engl J Med. 2020;382(13):1278-1280.

9. Chinazzi M, Davis JT, Ajelli M, et al. The effect of travel restrictions on the spread of the 2019 novel coronavirus (COVID-19) outbreak. Science. 2020;368(6489):395-400.

10. Holshue ML, DeBolt C, Lindquist S, et al. First case of 2019 novel coronavirus in the United States. N Engl J Med. 2020; 382(10):929-936.

11. Adalja AA, Toner E, Inglesby TV. Priorities for the US health community responding to COVID-19. JAMA. 2020;323(14): 1343-1344.

12. Parodi SM, Liu VX. From containment to mitigation of COVID-19 in the US. JAMA. 2020;323(15):1441-1442.

13. Burke JF, Chan AK, Mummaneni V, et al. Letter: The coronavirus disease 2019 global pandemic: a neurosurgical treatment algorithm. Neurosurgery. 2020;87(1):E50-E56.

14. Rubin EJ, Baden LR, Morrissey S, Campion EW. Medical journals and the 2019-nCoV outbreak. N Engl J Med. 2020; 382(9):866.

15. Okoli C, Pawlowski SD. The Delphi method as a research tool: an example, design considerations and applications. Inf Manage. 2004;42(1):15-29.

16. Eubank BH, Mohtadi NG, Lafave MR, et al. Using the modified Delphi method to establish clinical consensus for the diagnosis and treatment of patients with rotator cuff pathology. BMC Med Res Methodol. 2016;16(1):56.

17. Centers for Disease Control. Coronavirus (COVID-19). Accessed July 8, 2020. https://www.cdc.gov/coronavirus/2019nCoV/index.html

18. American College of Surgeons. COVID-19. Clinical issues and guidance. Accessed July 8, 2020. https://www.facs.org/ about-acs/covid-19/information-for-surgeons

19. Gostin LO. Medical countermeasures for pandemic influenza: ethics and the law. JAMA. 2006;295(5):554-556.

20. Fisher CG, DiPaola CP, Ryken TC, et al. A novel classification system for spinal instability in neoplastic disease: an evidence-based approach and expert consensus from the Spine Oncology Study Group. Spine (Phila Pa 1976). 2010; 35(22):E1221-E1229.

21. Ti LK, Ang LS, Foong TW, Ng BSW. What we do when a COVID-19 patient needs an operation: operating room preparation and guidance. Can J Anaesth. 2020;67(6):756-758.

22. Murthy S, Gomersall CD, Fowler RA. Care for critically ill patients with COVID-19. JAMA. 2020;323(15):1499-1500.

23. Chopra V, Toner E, Waldhorn R, Washer L. How should U.S. hospitals prepare for coronavirus disease 2019 (COVID-19)? Ann Intern Med. 2020;172(9):621-622.

24. Sangji NF, Bohnen JD, Ramly EP, et al. Derivation and validation of a novel Emergency Surgery Acuity Score (ESAS). J Trauma Acute Care Surg. 2016;81(2):213-220.

25. Ziewacz JE, Berven SH, Mummaneni VP, et al. The design, development, and implementation of a checklist for intraoperative neuromonitoring changes. Neurosurg Focus. 2012; 33(5):E11.

26. Conley DM, Singer SJ, Edmondson L, et al. Effective surgical safety checklist implementation. J Am Coll Surg. 2011; 212(5):873-879.

27. Haynes AB, Weiser TG, Berry WR, et al. A surgical safety checklist to reduce morbidity and mortality in a global population. N Engl J Med. 2009;360(5):491-499.

28. Weiser TG, Haynes AB, Dziekan G, et al. Effect of a 19-item surgical safety checklist during urgent operations in a global patient population. Ann Surg. 2010;251(5):976-980.

29. Wiegmann DA, ElBardissi AW, Dearani JA, et al. Disruptions in surgical flow and their relationship to surgical errors: an exploratory investigation. Surgery. 2007;142(5):658-665.

30. Berger MS, Wachter RM, Greysen SR, Lau CY. Changing our culture to advance patient safety: the 2013 AANS Presidential Address. J Neurosurg. 2013;119(6):1359-1369.

31. Lau CY, Greysen SR, Mistry RI, et al. Creating a culture of safety within operative neurosurgery: the design and implementation of a perioperative safety video. Neurosurg Focus. 2012;33(5):E3.

\section{Disclosures}

Dr. P. Mummaneni reports being a consultant for Globus, DePuy Synthes, and Stryker; having direct stock ownership in Spinicity/ISD; receiving support of non-study-related clinical or research effort from ISSG, AO Spine, and the NREF; and receiving royalties from DePuy Synthes, Thieme Publishers, and Springer Publishers. Dr. Chan reports support of non-studyrelated clinical or research effort from Orthofix Medical, Inc. Dr. Sosa reports being on the data monitoring committee of the Medullary Thyroid Cancer Consortium Registry supported by GlaxoSmithKline, Novo Nordisk, Eli Lilly, and AstraZeneca; 
and institutional research funding support of non-study-related clinical or research effort from Exelixis and Eli Lilly. Dr. Berven reports being a consultant for Medtronic, Stryker, Medicrea, Innovasis, and Integrity; receiving royalties from Medtronic and Stryker; having ownership in Green Sun Medical and Providence Medical; and being on the JNS Editorial Board. Dr. Goldberg reports being an advisor to Keyssa, Inc. Dr. Vail reports receiving royalties from DePuy and having ownership in Hyalex.

\section{Author Contributions}

Conception and design: PV Mummaneni, Burke, Chan, Berger. Acquisition of data: PV Mummaneni, Burke, Chan, Sosa,

Lobo, VP Mummaneni, Antrum, Berven, Conte, Doernberg,

Goldberg, Hess, Hetts, Josephson, Kohi, Ma, Mahadevan, Murr, Narayana, Roberts, Stoller, Theodosopoulos, Vail, Weinzholz,
Gropper, Green, Berger. Analysis and interpretation of data: PV Mummaneni, Burke, Chan. Drafting the article: PV Mummaneni, Burke, Chan, Molinaro, Berger. Critically revising the article: all authors. Reviewed submitted version of manuscript: all authors. Approved the final version of the manuscript on behalf of all authors: PV Mummaneni. Statistical analysis: Burke, Molinaro. Study supervision: PV Mummaneni, Berger.

\section{Correspondence}

Praveen V. Mummaneni: University of California, San Francisco, CA.praveen.mummaneni@ucsf.edu. 